NOTICIAS Y COMENTARIOS 
204 


\title{
LA CONSERVACIÓN DE LOS PAISAJES ATERRAZADOS EN LA TOSCANA: ENTRE LA UTOPÍA Y LA REALIDAD ${ }^{1}$
}

\author{
María Hernández Hernández
}

\section{RESUMEN}

Las notables transformaciones que se producen tras la Segunda Guerra Mundial en la estructura socio-económica italiana, se han traducido en profundos cambios en la agricultura tradicional. Hecho que ha generado notables degradaciones ambientales. Áreas que, con la difusión de la nueva filosofía ambiental, pueden adquirir un nuevo valor. Así, frente a la función productiva, en crisis, estos espacios podrían enarbolar la bandera del valor ambiental, cultural o paisajístico. Sin embargo, para lograrlo, es necesario la adopción de una serie de disposiciones tendentes a la conservación de la sistematización hidráulica y agraria dieciochesca, su principal atractivo. Sin embargo, ésta choca con una serie de dificultades entre las que destacan la escasez de financiación, la lentitud en aplicar la legislación o el carácter contradictorio de ciertas normativas.

Palabras clave: ordenación tradicional de las laderas, degradación ambiental, nuevos usos del territorio, conservación de la sistematización tradicional, dificultades para su conservación.

1 El presente artículo es fruto de la investigación realizada el pasado febrero en la región de Toscana. Tarea que no se podría haber desarrollado sin la inestimable ayuda de los profesores R. Landi (Instituto de Agronomía), M. Azzari y F. Canigiani (Instituto de Geografía) de la Universidad de Florencia, D. Croce (Dipartimento di Geografía G. Morandini, Università di Padova) o la colaboración de los investigadores del C.E.D.I.P. y del Istituto Sperimentale per la difesa dil suolo. 
Economic problems, connected with market changes and technical needs produced in Italy deep changes in the agriculture: land management systems of sloping areas are given up. Abandon that has produced remarkable environment problems. Areas which have a new worth as resault of the new environment phylosophy (forestry, turist or sport activity, part-time agriculture). Nevertheless, if we want to develop it, it is necessary investiment to maintenance land and use management. Polity that face serious problems: short finance, slowness to adjundge laws or the contradictory disposition of same precept.

Key words: traditional land management of sloping areas, environmet impact, new territorial use, traditional, land management systems mantenance, problems to preserve it.

\section{Introducción}

Los principios sobre la conservación del suelo tienen su origen en la cuenca mediterránea. En los albores de la Historia se realizan grandes obras hidráulicas de bonificación y de defensa, basta recordar «l'inversione del corso del Clanis, il taglio della Gonfolina, le fosse filistine, la Cloaca massima e i $30 \mathrm{~km}$ di cunicoli fognati di Vejo»². Los postulados básicos de la ordenación hidráulico-agraria aparecen ya recogidos en las obras de Esiodo, Catón, Virgilio o Columela. Técnicas que adquieren una gran difusión a mediados del siglo dieciocho cuando el sistema de cultivo tradicional (rittochino) caracterizado por trabajar la tierra siguiendo las líneas de máxima pendiente es sustituido por una ordenación basada en la construcción de campos gradados.

Métodos que fueron iniciados por G.B. Landeschi, párroco de San Miniato y A. Testaferrata, administrador de Meleto. La cuna de sistematización hidráulicoagraria es, por tanto, el triángulo que tiene por vértices Florencia, Pisa y Siena. Ordenación que es reflejo de una sociedad, obra de unos hombres que tuvieron que adaptarse a unas condiciones edáficas poco aptas para el cultivo y que, por tanto, merecen conservarse. Oliva ya en 1948 escribía «se nel mondo agronomico fosse giuridicamente ammessa la conservazione ed il rispetto dei monumenti nazionali. Meleto sarebbe il primo caso proponibile ${ }^{3} \gg$.

Paisaje colinar que hasta el final de la segunda guerra mundial se caracterizó por la existencia de pequeños predios, difusión de la coltura promiscua, uso de la

2 LANDI, R.: «La conservazione dei terreni nelle richerche e negli ordenamenti didattici della Facoltà di Agraria», Riv. di Storia dell'Agricoltura, Accademia economico-agraria dei Georgofili, junio 1985.

3 OLIVA, A.: Le sistemazioni idraulico-agrarie, Barbera, Firenze, 1948. 
tracción animal y abundancia de mano de obra. Técnicas que permitían un trabajo superficial del suelo que unido a la constante reparación de los numerosos canales de drenaje existentes, permitían la conservación de un espacio que era más una construcción del hombre que un paisaje natural ${ }^{4}$.

Sectores que experimentarán notables cambios paisajísticos como consecuencia de las transformaciones socio-económicas (éxodo rural, reducción de los activos primarios, introducción de maquinaria pesada, abandono de tierras, empleo de técnicas inadecuadas, etc.) que se producen al finalizar la Segunda Guerra Mundial. Mutaciones que se manifiestan en los trasformación en los usos del suelo: espacios dedicados tradicionalmente a actividades primarias, son ocupados bien por instalaciones industriales, por la expansión urbana o por creación de núcleos turísticos. Y asociados a este proceso, la intensificación de los procesos erosivos entre los que destacan la proliferación de cárcavas denominadas en la zona calanchi, el afloramiento de terrenos con alto contenido salino (bianchane o crete senese) como consecuencia de la acción de denudación que ejercen las aguas de lluvia, los deslizamientos y desplomes o el aumento de la velocidad y del transporte de materiales sólidos en las ondas de crecida.

\section{Legislación y estudios sobre la ordenación agraria tradicional}

En este último siglo la conservación del suelo y la sistematización hidráulicoagraria de las laderas ha sido objeto de numerosos estudios, convenios, seminarios, y de intervenciones legislativas de carácter nacional y regional. A continuación indicaremos los más relevantes.

Numerosos congresos se han celebrado durante la presente centuria para analizar esta problemática. El primer coloquio nacional fue realizado en Roma en 1935, patrocinado por el Sindicato de Técnicos Agrarios. Posteriormente en 1939 y organizado por la Academia dei Georgofili se celebró el Congresso per le Sistemazioni idraulico-agrarie. Temáticas similares se analizaron en las reuniones que se llevaron a cabo en la década de los 40 entre las que cabe mencionar el Convegno di aggiornamento (Roma, 1942) convocado por el Instituto Nacional de Técnica Agraria o el Convenio agrario italo-americano (Florencia, 1946). Seminarios que continúan organizándose en los años posteriores como el organizado por el A.N.B.I, (La protezione del suolo e la regolaziones delle acque, Roma, 1967) y que se intensificarán en la década de los ochenta como reflejo de una mayor preocupación por los temas ambientales. Mereciéndose destacarse los siguientes: Convenio financiado por el C.N.R sobre la conservación del suelo (Roma, 1982), el XVII

4 Nueva ordenación de las laderas que es analizada en LANDESCHI, G.B: Interpretación del paisaje agrario de la Toscana en el siglo XVIII (Saggi di Agricoltura), Estudio preliminar y traducción María Hernández Hernández, Instituto Universitario de Geografía, Universidad de Alicante, 1994. 
Congreso della S.I.A (Il ruolo dell'Agricoltura nella conservazione del suelo, Florencia, 1983), o el celebrado en la misma ciudad en 1988 con el título La sistemazione della collina per la difesa del suolo e la tutela dell'ambiente, o los seminarios celebrados en Meleto en 1988 y 1991 (L'esperienza di Ridolfi y Sistemazioni idrauliche delle colline toscane).

Ordenación de estos espacios colinares que ha sido siempre considerada como una obra que por sus implicaciones económicas y sociales requiere la intervención del estado. Así han sido emitidos diversos reglamentos desde el Ministerio de Agricultura que interesan a estos sectores y donde se defiende la necesidad de este tipo de ordenación (Primera circular del Ministerio dirigida a los Inspectores Agrarios), o la creación de organismos relacionados con el tema entre los que destacan la fundación en 1967 en Florencia del Istituto Sperimentale per la Difesa e la Conservazione del Suolo, o la creación dentro del Ministerio de Agricultura de un Servizio Nazionale per la conservazione e la difesa del suolo.

Numerosas directivas comunitarias y leyes nacionales o regionales defienden cada vez con mayor vehemencia la necesidad de salvaguardar este patrimonio ambiental y paisajístico. Disposiciones que generalmente se insertan dentro de una unidad de cuenca (bacino idrografico) en los que se ha elaborado un Piano generale di bonifica e di tutela del territorio rurale.

Merece asimismo destacarse el reglamento de la CEE 2078/92 y la ley del Ministerio de Agricultura de 1 de febrero de 1993 con la que se aplica el mencionado reglamento, que prevee programas zonales regionales con una duración quinquenal y entre cuyas intervenciones propone medidas de salvaguardia y mejora del ambiente privilegiando los territorios montanos y colinares por su mayor riesgo de erosión. Sobre la declaración de zonas protegidas, cabe mencionar la ley Galasso, (L.R. 52/82 de 29 de junio de 1985) y las posteriores reglamentaciones regionales.

\section{La conservación de la ordenación agraria tradicional: una utopía}

Sin embargo, observando la situación en que se encuentra la mayor parte del agro toscano, debemos afirmar que, a pesar de los números estudios, congresos y leyes, éstos han sido en gran parte ignorados. Así, por ejemplo, la mencionada Ley Galasso tiene todavía un marcado carácter introductivo, y escasa eficacia inmediata, al configurarse como un plan regional de tutela ambiental graduado en el tiempo y que no se traducirá necesariamente en la institucionalización de parques y reservas naturales ${ }^{6}$.

5 BARBIERI, G.: Legge Galasso, direttive CEE e aree protette in Toscana, Istituto di Geografía, Università di Firenze, 1987.

6 CIAMPI, G.: «Osservazioni su alcune scelte normative di rilievo ambientale interesanti i demani universali» Atti del'Istituto di Geografía, 15, Università di Firenze, 1990. 
Progresivo desinterés por las labores de mantenimiento cara a la protección del suelo, y defensa de las sistematizaciones agrarias tradicionales que hay que relacionar con ciertos procesos. Es evidente que la urbanización salvaje que han experimentado algunos espacios ha conducido a una ocupación de los terrazgos agrícolas más aptos; pero también es innegable que la financiación a favor de la protección del suelo ha sido drásticamente reducida ${ }^{7}$. Proceso favorecido asimismo por ciertas normativas que no conceden al problema su legítimo valor o su carácter confuso y contradictorio ${ }^{8}$, o la lentitud con que son aplicadas ${ }^{9}$. Mención destacada reviste también la decadencia de la conciencia cultural de la población en lo que se refiere a la relación agricultura-medio ambiente.

Sensibilidad que era un patrimonio acumulado durante siglos en que los hombres debían adaptarse a la naturaleza para poder sobrevivir, pero sin generar graves impactos y que ha sido denominada por Mazzocchi Alemanni como «coscienza sistematoria». Conciencia que en parte ha desaparecido como reflejo de una necesidad de los agricultores de producir abaratando costes para poder ser competitivos dentro de una economía de mercado cada vez más feroz lo que implicaba la utilización de tecnología altamente contaminante, frente a unas técnicas más extensivas pero más adecuadas desde el punto de vista ambiental. Dilema que continua en la actualidad en la que desde la Comunidad se propugna por la adopción de practicas conservacionistas, las cuales en la actualidad no son rentables desde una óptica meramente economicista.

Escasa atención a estos espacios colinares que se relaciona con una serie de hechos entre los que destaca que este tipo de agricultura, donde aún se practica, es casi siempre una actividad marginal. Además, los daños generados por el abandono, descuido de estas prácticas afectan a pequeñas y medianas propiedades, apareciendo distribuidas entre un número elevadísimo de propietarios de ahí la escasa

7 Es evidente la exigüidad de las cantidades presupuestadas por la Ley 500/92 que ha asignado únicamente 350.000 millones de liras en 1993 y 250.000 para el 1994 frente a los 350.000 de 1955 . Hecho que llama la atención ante la importancia que a la defensa del suelo le concedía la ley 183 de 18 de mayo de 1989.

8 La aplicación de ayudas destinadas a favorecer el abandono definitivo de ciertos cultivos puede intensificar los problemas relacionados con la conservación del suelo. Es evidente, que es difícil pedir que ciertos agricultores mantengan la sistematización colinar en tierras que han dejado de cultivar acogiéndose a las normativas comunitarias, sobre todo cuando éstas son confusas y contradictorias. Así, en la solicitud de concesión del DM 35 de 8 de enero de 1990, por ejemplo, el solicitante, en el caso de superficies que se van a dejar incultas o con posibilidad de rotación de cultivos, se obliga a «creare o mantenere un'adeguata copertura vegetale, in particolare per prevenire l'erosione o la lisciviazione dei nitrati». Sin embargo, la misma directiva exige «effettuare le necessarie lavorazioni meccaniche del suolo, in particolare per conservare la riserva idrica e per lottare contro le erbe infestanti».

9 A modo de ejemplo, podemos indicar que fue en noviembre de 1991 cuando la autorità di bacino, las regiones y las provincias autónomas, se pusieron de acuerdo acerca de la prioridad en las actuaciones a financiar para el período 1989-93. 
atención que reciben de la opinión pública, de los medios de comunicación e incluso de la administración.

\section{Disposiciones a adoptar cara a su conservación}

Sectores que manteniendo esta sistematización, se encontrarían en una situación óptima ante las nuevas demandas de una sociedad que cada vez valora más los espacios verdes, el agua limpia, el aire respirable, los paisajes agradables. Ante estas tendencias, el territorio rural colinar podría revalorizarse y en este proceso, unido a la función productiva aparecen otras quizás más importantes como son las de orden ambiental, cultural y paisajístico. Así, estos espacios podrían dedicarse a actividades relacionadas con el agroturismo tan difuso en esta región, áreas verdes en las proximidades de las grandes ciudades, agricultura biológica o a tiempo parcial, etc. Su supervivencia pasa, por tanto, por la adopción de una «calidad», frente a la «cantidad», con la que, dados sus elementos definidores (parcelas de reducida extensión, pendientes elevadas, etc.), no puede competir con la agricultura que se practica en los valles.

A pesar de los obstáculos descritos en epígrafes anteriores, debemos postular la potenciación de disposiciones ya adoptadas o la introducción de otras nuevas para intentar abordar el problema desde una óptica práctica y que el mantenimiento de estos espacios sea más real. Soluciones entre las que cabría citar:

- establecer para cada situación pedoclimática y cultural técnicas precisas y lo más simples posibles para la conservación de los elementos definidores de la ordenación agraria tradicional.

- necesidad de formar especialistas en este sector y sobre todo la difusión en amplios estratos de la sociedad de lo beneficioso que es conservar estos paisajes como reflejo de una sociedad, de un tiempo pasado, de una coscienza sistematoria hoy casi olvidada.

- promover con incentivos a fondo perdido y desgravaciones fiscales la reparación de los elementos definidores de esta sistematización, pues su mantenimiento representan en la actualidad una labor técnica y una carga económica que la escasa producción de las áreas colinares no pude soportar.

- asegurar la presencia del hombre incluso en las áreas más marginales como garantía de equilibrio ecológico. Poblaciones locales que serán las encargadas de su gestión y cuidado, es decir, frente a la considerada «tutela pasiva» basada en medidas sujetas a obligaciones legales, se prevén disposiciones de «tutela activa» gracias a la adopción de planes de desarrollo socio-económico. Se trata, por tanto, además de evitar las tensiones y los conflictos que localmente ralentizan la adopción de disposiciones conservativas, ofrecer a la población compensaciones por las eventuales sustracciones de terrenos a los usos no compatibles, beneficios económicos alternativos para garantizar condiciones de permanencia dignas. 
- Introducción de nuevas actividades acordes con el medio ambiente y que contribuyan a fijar población. Es evidente que en la actualidad a la agricultura no se le pide el ser una actividad cuya única finalidad sea la de resolver los problemas alimenticios de la población, sino que se le demanda que sea respetuosa con el entorno. Sin embargo, si se aceptan estos presupuestos, es evidente que los campesinos deberán recibir alguna compensación económica. En este sentido hemos de valorar en su justa medida la existencia de la figura de agricultor a tiempo parcial que ha contribuido de una manera efectiva a una adecuada conservación de algunos de estos espacios agrícolas tradicionales.

- favorecer la reforestación de aquellos ámbitos colinares donde la agricultura ha sido abandonada y que están siendo recolonizados por especies vegetales naturales. Espacios muchos de ellos con clara vocación forestal y que fueron puestos en cultivo en épocas en que el aumento en la presión demográfica exigía roturar nuevas tierras.

- individualización de zonas protegidas. En la década de los 80 tras una investigación llevada a cabo de manera coordinada por la Región y dos institutos universitarios (Geografía y Arquitectura) se singularizaron posibles áreas a proteger. Así, se han identificado 166 áreas «protegidas», lo que representa el $30 \%$ del territorio de la Toscana. Sin embargo, no se trata de confeccionar un mero elenco de estas zonas, sino de realizar una adecuada ordenación del territorio en la cual queden claramente delimitados los usos compatibles con estos territorios.

\section{Meleto: un ejemplo a perfeccionar, imitar y difundir}

Meleto es una hacienda de gran valor histórico: Ridolfí y su administrador Testaferra pusieron en práctica de manera experimental la sistematización colinar, iniciada por Landeschi y que posteriormente se difundió por toda la Toscana y sin la que no se puede comprender el desarrollo agrícola de dicha región en el ochocientos, bajo el auspicio de la dinastía de los Lorena. Villa donde se fundó una de las primeras escuelas prácticas de agricultura, destinada a la enseñanza de los jóvenes que posteriormente debían dirigir explotaciones agrarias. Su creación, por tanto, responde a uno de los objetivos ilustrados como era reducir la ignorancia tan extendida en el agro y que se traducía en notables pérdidas económicas en una época en el que el crecimiento demográfico y el reinicio de los intercambios comerciales exigía una mayor producción.

Elementos definidores de esta ordenación (taludes, canales de drenaje, etc.), especialmente la denominada «a spina» que han sido reparados, al menos los más cercanos a la villa, con el apoyo financiero del gobierno de la Toscana con el fin de conservarlo para estudiar estas técnicas y aplicarlas posteriormente para evitar pérdidas edáficas. Además, se ha constituido el «Museo Ridolfiano» con el que se 
pretende divulgar la protección ambiental tan íntimamente legada a las prácticas agrícolas de finales del setecientos.

Sin embargo, la tarea no ha hecho más que iniciarse ya que junto a estos espacios más o menos conservados, existen otros con graves problemas de deterioro como en Montalbano donde las laderas con muros de piedra seca muestran numerosos manifestaciones de la incisión de las aguas de lluvia. De modo que, podemos afirmar que si no se interviene pronto se verá irremediablemente alterado este patrimonio cultural.

En consecuencia, el ejemplo de Meleto debe ser imitado, no obstante, indicaremos que no se debe pensar únicamente en crear espacios protegidos gracias a las ayudas estatales, sino que se deben mantener ciertos usos que favorezcan su conservación, que fijen la población, ya que sin estos presupuestos es imposible pensar en una adecuada conservación.

\section{6. Áreas verdes y parques territoriales en la Toscana: Monte Morello, un parque periurbano para el área metropolitana florentina}

Afirma la profesora F. Canigiani que «es un hecho constatado que la política de los parques ha registrado un evidente estancamiento al menos a nivel estatal ${ }^{10} \gg$. Evidentemente esta falta de operatividad es debida sobre todo al hecho de que éstos «ocupan una posición relativamente baja en la escala de prioridades de la población italiana ${ }^{11} \mathrm{y}$, por tanto, a la falta de una actitud cultural difundida de respeto hacia la conservación de la naturaleza y el ambiente. Hecho en parte que se ha visto favorecido por la conflictividad de competencias surgidas entre las regiones y el estado.

Aspectos estos que se manifiestan en este parque que generalmente se identifica con el macizo del Monte Morello ${ }^{12}$, pero que es ampliable a una zona más vasta conocida como Colli Alti fiorentini. Espacio donde el paisaje agrario característico de la colina toscana asume todas sus peculiaridades y junto a ésta la presencia monumental. Estos últimos cubren un largísimo período de la historia: época protohistórica (municipios de Quinto, Palastreto e Castellina), las tumbas etruscas en Mula y Montagnola, los castillos y casas-torre medievales, las villas renacentistas y del setecientos (ejemplo, la Villa Demidoff donde el C.E.D.I.P. ha instalado

10 ANCONA, L. y CANIGIANI, F. «L'Italia «protetta», Atti dell'Istituto di Geografía $n^{\circ} 14$, Università di Firenze, 1989, p. 13.

11 SCHMIDT DI FRIEDBERG, P.: «La percezione dei parchi da parte delle comunità come base per l'istituzione e la gestione di essi», en A.A.V.V.: Convegno sul tema: Parchi e aree protette in Italia, Consiglio Nacionale delle Richerche, 1985, p. 301.

12 DI PIETRO, G.F.: «I caratteri peculiari del Parco di Monte Morello», Atti del I Convegno sullo stato dell'ambiente a Sesto Fiorentino, Firenze, 1989. 
su sede con el fin de agrupar todas las iniciativas de cara a la política de zonas protegidas) o las iglesias parroquiales y los monasterios ${ }^{13}$.

Espacio que ha adquirido el carácter de área protegida con la L.R. 29/6/82 no 52 y la 8/8/1985, n $^{\circ} 431$ en la que se recogen las disposiciones a adoptar para la tutela de zonas de particular interés ambiental. Legislación que, pretende delimitar las actividades en un espacio donde la presión ejercida por los usos urbanos es cada vez más intensa. Proceso paralelo a la marginalidad cada vez más evidente de la agricultura.

Sin embargo, pronto surgen los conflictos al delimitar las competencias entre los distintos niveles administrativos. Al perímetro y usos establecidos por el gobierno regional, hay que unir las previsiones de cada unos de los municipios afectados, que aparecen recogidas en los diferentes Piani Regolatori Generali ${ }^{14}$. Son los ayuntamientos de Fiesole y Sesto Fiorentino los que han adoptado una posición más coherente en su defensa con la adopción de un P.R.G para las zonas agrícolas colinarias, frente a la ausencia de una normativa concreta para estos espacios de los municipios de Vaglia, Calenzano y Florencia. No obstante, hemos de indicar que la «conservación» de esta área se debe más a voluntad propia de los habitantes que al respeto de esta normativa: en un espacio donde el hábitat disperso aparece tan difundido, unido a su carácter metropolitano, es muy frecuente encontrar en los pequeños huertos familiares e incluso en los propios jardines de la viviendas, las elementos definidores del paisaje toscano, que son conservados por sus moradores al encontrarse dentro de sus propiedades.

\section{Bibliografía}

ANCONA, L. y CANIGIANI, F.: «La Toscana «protetta», Atti dell'Istituto di Geografía $n^{\circ}$ 14, Università di Firenze, 1989.

ATTI DEL CONVEGNO: Sistemazioni idraulico-agrarie delle colline toscane, Meleto, 1991.

AZARRI, M. et al.: «Le ragioni dei Parchi e l'Italia «protetta»: iniziative e problemi», Atti dell'Istituto di Geografía n ${ }^{\circ}$ 15, Università di Firenze, 1990.

CHISCI, G.: Influence of change in land use ad management on the acceleration of land degradation phenomen in Appennino hilly areas, Casena, 1986.

GASPARINI, M.: Evoluzione delle sistemazioni idraulico-agrarie nelle terre declivi. Difesa e conservazione del suolo dalle erosione idrogeologiche, Ins. Tecn e Prop. Agr, Roma, 1970.

LANDI, R.: «Regimazione idraulico-agraria e conservazione dell suolo, Riv. di Agronomia XVIII, 1984.

13 REGIONE TOSCANA: Il sistema regionale delle aree verdi, CEDIP, Villa Demidoff, Firenze, 1990 .

14 RALLO, A: «Diritti di uso civico e pianificazione urbanistica», Riv. Giuridica dell'edilizia, XXVIII, 1985, parte II, pp. 195-211. 